\title{
An Elliptical Galaxy Luminosity Function and Velocity Dispersion Sample of Relevance for Gravitational Lensing Statistics
}

\author{
Yu-Chung N. Cheng ${ }^{1}$ and Lawrence M. Krauss ${ }^{2}$ \\ Department of Physics, Case Western Reserve University, 10900 Euclid Ave., \\ Cleveland, $\mathrm{OH}$ 44106-7079, USA
}

\begin{abstract}
We have selected 42 elliptical galaxies from the literature and estimated their velocity dispersions at the effective radius $\left(\sigma_{R_{\mathrm{e}}}\right)$ and at 0.54 effective radii $\left(\sigma_{0.54 R_{\mathrm{e}}}\right)$. We find by a dynamical analysis that the normalized velocity dispersion of the dark halo of an elliptical galaxy $\sigma_{\mathrm{DM}}$ is roughly $\sigma_{R_{\mathrm{e}}}$ multiplied by a constant, which is almost independent of the core radius or the anisotropy parameter of each galaxy. Our sample analysis suggests that $\sigma_{\mathrm{DM}}^{*}$ lies in the range $178-198 \mathrm{~km} \mathrm{~s}^{-1}$. The power law relation we find between the luminosity and the dark matter velocity dispersion measured in this way is $\left(L / L^{*}\right)=\left(\sigma_{\mathrm{DM}} / \sigma_{\mathrm{DM}}^{*}\right)^{\gamma}$, where $\gamma$ is between $2-3$. These results are of interest for strong gravitational lensing statistics studies.

In order to determine the value of $\sigma_{\mathrm{DM}}^{*}$, we calculate $\mathrm{M}_{\mathrm{B}_{\mathrm{T}}(0)}^{*}$ in the same $\mathrm{B}_{\mathrm{T}}(0)$ band in which $\sigma_{\mathrm{DM}}^{*}$ has been estimated. We select 131 elliptical galaxies as a complete sample set with apparent magnitudes $\mathrm{B}_{\mathrm{T}}(0)$ between 9.26 and 12.19. We find that the luminosity function is well fitted to the Schechter form, with parameters $\mathrm{M}_{\mathrm{B}_{\mathrm{T}}(0)}^{*}=-19.66+5 \cdot \log _{10} h \pm 0.30, \alpha=0.15 \pm 0.55$, and the normalization constant $\phi^{*}=(1.34 \pm 0.30) \times 10^{-3} h^{3} \mathrm{Mpc}^{-3}$, with the Hubble constant $\mathrm{H}_{\mathrm{o}}=100 \mathrm{~h} \mathrm{~km}$ $\mathrm{s}^{-1} \mathrm{Mpc}^{-1}$. This normalization implies that morphology type E galaxies make up $(10.8 \pm 1.2)$ per cent of all galaxies.
\end{abstract}

Key words: cosmology: gravitational lensing, galaxies: fundamental parameters, galaxies: halos, galaxies: kinematics and dynamics, galaxies: luminosity function, methods: statistical

PACS: 98.80, 95.30.S

$\overline{1}$ Also in The MRI Institute for Biomedical Research, E-mail address: yxc16@po.cwru.edu

2 Also in Department of Astronomy, E-mail address: krauss@theory1.phys.cwru.edu 


\section{INTRODUCTION}

The search for the dark matter plays a central role in modern astrophysics and cosmology. A key factor in constraining the cosmic abundance of dark matter is to measure rotation curves or velocity dispersions of galaxies. Because spiral galaxy rotation curves provide sensitive constraints on dark matter, it is common to measure velocity dispersions in these systems. However, there are fewer available data sets of velocity dispersions for elliptical galaxies.

At the same time, the characteristic velocity dispersion of elliptical galaxies is a very important parameter in the statistical study of strong gravitational lensing, because the optical depth for lensing by galaxies is proportional to the fourth power of the velocity dispersion (Turner, Ostriker \& Gott 1984). The value of the normalized dark matter velocity dispersion for luminous elliptical galaxies that should be used in the strong gravitational lensing analysis is still under some debate. For example, in 1984 Turner et al. (1984) suggested that one should use directly observed velocity dispersions, and multiply them by $\sqrt{3 / 2}$ to determine dark matter velocity dispersions, as suggested by the virial theorem. However, Kochanek $(1993,1994)$ has argued using dynamical models that one should simply use the measured velocity dispersions as the dark matter velocity dispersions for the lensing calculation, without any $\sqrt{3 / 2}$ correction. However, even in this case, one must still estimate the characteristic dark matter velocity dispersion for ellipticals, and this depends on one's assumptions about the elliptical galaxy luminosity function (Cheng \& Krauss 2000). Moreover, both Kochanek $(1993,1994)$ and Turner et al. (1984) focussed on the singular isothermal sphere (SIS) model in their gravitational lensing analysis. This model is clearly a very rough approximation to actual galaxies.

In this paper, our goal is to re-examine both the appropriate luminosity function and velocity dispersions for use in lensing statistics analyses. In our analysis we consider a finite core model, and consider the self-consistent determination of the velocity dispersion and luminosity function for ellipticals. This analysis is relevant even if the core radius is quite small, or zero. To be consistent we calculate the luminosity function in the same band in which we estimate velocity dispersions.

We choose the Hubble constant $\mathrm{H}_{\mathrm{o}}=100 h \mathrm{~km} \mathrm{~s}^{-1} \mathrm{Mpc}^{-1}$ throughout. We investigate the luminosity function in Section 2. In section 3 we review our investigation of the theoretical determination of the dark matter velocity dispersion in galaxies with core radii using dynamical models with luminosity profiles discussed by Jaffe (1983) and Hernquist (1990). In Sections 3.1 and 3.2, we will describe our main results appropriate for use in lensing studies, including normalized velocity dispersions and their power law relations with the luminosity. 


\section{THE LUMINOSITY FUNCTION}

The appropriate value of $\mathrm{M}^{*}$ in a Schechter formulation (1976) of the luminosity function will depend on which magnitude system one is measuring, so that there is no unique value of $\mathrm{M}^{*}$. In this paper, we will investigate the shape of the Schechter luminosity function for a sample of elliptical galaxies in the $\mathrm{B}_{\mathrm{T}}(0)$ band, as defined in de Vaucouleurs et al. (1991) (hereafter RC3). In order to calculate the luminosity of each galaxy in the $\mathrm{B}_{\mathrm{T}}(0)$ band, we choose the distance of each galaxy from Faber et al. (1989) (hereafter 7s), and the apparent magnitude $\mathrm{B}_{\mathrm{T}}(0)$ of each galaxy from RC3. We find 415 galaxies in this set. However, we need to obtain a complete magnitude limited sample set to calculate the luminosity function (Loveday et al. 1992). Based on the discussion in 7s, the elliptical galaxies in 7s are complete to apparent magnitude 11.89. However, the RC3 catalogue was published after the appearance of $7 \mathrm{~s}$. Therefore, we consider galaxies with the apparent magnitudes given in $7 \mathrm{~s}$ less than or equal to 11.89, and then check out their corresponding apparent magnitudes in RC3. We find the dimmest (or the largest number) apparent magnitude among these galaxies is 12.19. Thus, we use 12.19 as the cutoff apparent magnitude in RC3, and select those galaxies in 7s whose corresponding apparent magnitudes in RC3 are less than 12.19. There are 131 galaxies in the final sample set. We then determined the luminosity function of this sample as described below.

\subsection{STY parametric maximum likelihood method}

We use here STY maximum likelihood method (Sandage, Tammann \& Yahil 1979). There are some advantages to use this method, as discussed in Efstathiou, Ellis \& Peterson (1988) (hereafter EEP). Consider the following probability

$$
p_{i} \propto \frac{\phi\left(L_{i}\right)}{\int_{L_{\min }\left(d_{i}\right)}^{L_{\max }\left(d_{i}\right)} \phi(L) d L}
$$

with Schechter (1976) function

$$
\phi\left(L_{i}\right)=\frac{1}{L^{*}} \phi^{*}\left(\frac{L_{i}}{L^{*}}\right)^{\alpha} \exp \left(-\frac{L_{i}}{L^{*}}\right)
$$

This is the probability to find a galaxy at distance $d_{i}$ with luminosity $L_{i}$ in the magnitudelimited sample set. $L_{\max }\left(d_{i}\right)$ and $L_{\min }\left(d_{i}\right)$ are calculated by using the distance $d_{i}$ (in unit of $h^{-1} \mathrm{Mpc}$ ) and the brightest and the dimmest apparent magnitudes among the whole magnitude-limited sample set. For example, the brightest apparent magnitude in our sample set is 9.26 , so $L_{\max }\left(d_{i}\right) / L^{*}$ will be equal to $d_{i}^{2} \cdot 10^{10+0.4\left(\mathrm{M}^{*}-m_{\max }\right)}$, with $m_{\max }=9.26$. The likelihood function is $\mathcal{L}=\prod_{i=1}^{\mathcal{N}} p_{i}$, and $\mathcal{N}$ is the total number of galaxies in the sample set. 


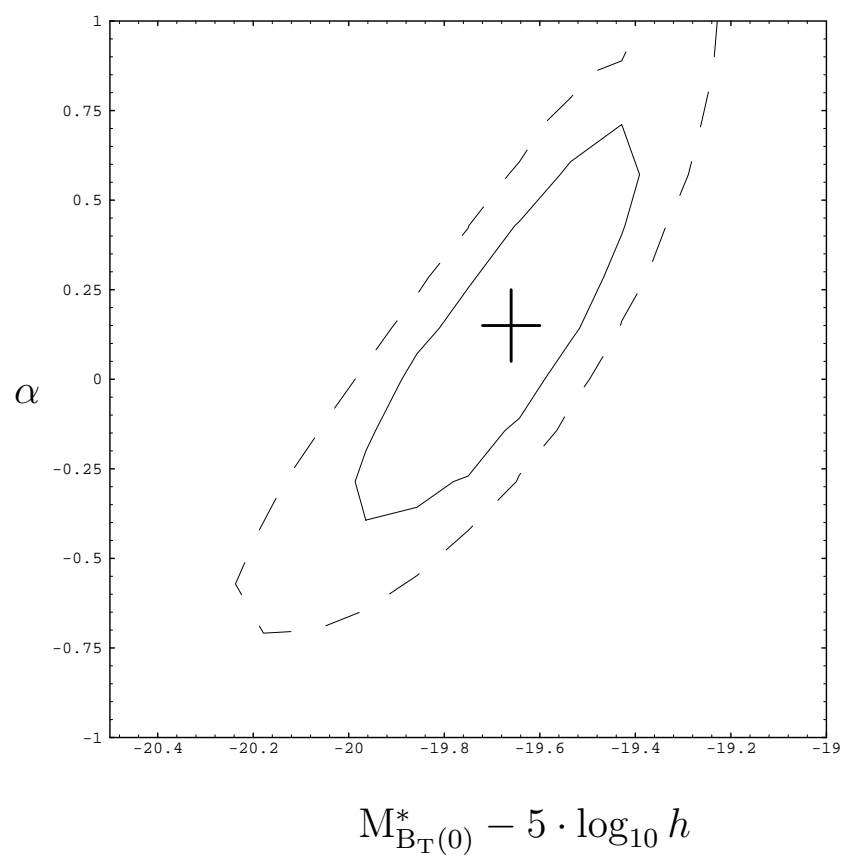

Fig. 1. Parameters, $\alpha$ and $\mathrm{M}_{\mathrm{B}_{\mathrm{T}}(0)}^{*}-5 \cdot \log _{10} h$, are fitted to the Schechter function by using STY maximum likelihood method. The best fit has been marked ' + .' The solid curve is the $1 \sigma$ error contour, and the dash curve is the 95 per cent confidence level contour. The statistical method is discussed in the text.

We maximize the likelihood function $\mathcal{L}$ with two parameters $\alpha$ and $L^{*}$ (or $\mathrm{M}^{*}$ ). We do not additionally correct for a Malmquist bias because the average dispersion between different $\mathrm{B}_{\mathrm{T}}$ magnitudes in RC3 is small, and also because Faber et al. (1989) already corrected for the Malmquist bias when they estimated the final distances of the galaxies in $7 \mathrm{~s}$. From the maximum likelihood analysis, we get the parameters $\mathrm{M}_{\mathrm{B}_{\mathrm{T}}(0)}^{*}=-19.66+5 \cdot \log _{10} h_{-0.33}^{+0.27}$ and $\alpha=0.15_{-0.54}^{+0.57}(1 \sigma$ error) (Figure 1$)$.

Because of the use of two different samples to attempt to obtain a complete magnitude limited survey, one might be concerned that different choices of magnitude cutoff would produce different maximum likelihood estimates for the luminosity function parameters. We explored a variety of combinations in this regard, and found consistent results within the uncertainties quoted above. For example, if we consider galaxies whose apparent magnitudes are below the apparent magnitude threshold in $7 \mathrm{~s}$, then find the corresponding apparent magnitudes in RC3, and neglect one apparently anomalous faint galaxy in 7s with apparent magnitude 11.00 in RC3, then the next galaxy which is below the threshold in 7s has apparent magnitude 11.83 in RC3. If we use 11.82 as the cutoff apparent magnitude, then we will have 82 galaxies in the sample set, and then we get $\mathrm{M}_{\mathrm{B}_{\mathrm{T}}(0)}^{*}=-19.78$ and $\alpha=0.22$. Choosing a fainter cutoff, 11.98, we find $\mathrm{M}_{\mathrm{B}_{\mathrm{T}}(0)}^{*}=-19.74$ and $\alpha=0.00$ with 107 galaxies in the sample set. As claimed, these are within the uncertainties in our quoted value above. 


\subsection{Step-wise maximum likelihood method}

In order to examine the goodness of fit of the Schechter function to the data we utilize a Step-wise maximum likelihood method to fit the data (EEP). This method is basically to replace the integral in equation (1) with finite sum. Following presentation in EEP, we define

$$
\phi_{k}^{\prime}=\frac{\sum_{i=1}^{N} D e\left(L_{i}-L_{k}\right)}{\sum_{i=1}^{N} \frac{T h\left[L_{k}, L_{\min }\left(d_{i}\right), L_{\max }\left(d_{i}\right)\right]}{\sum_{j=1}^{N_{\mathrm{p}}} \phi_{j}^{\prime} \cdot T h\left[L_{j}, L_{\min }\left(d_{i}\right), L_{\max }\left(d_{i}\right)\right]}}
$$

where

$$
\begin{aligned}
& \phi_{k}^{\prime}(L) \equiv \phi^{\prime}\left(L_{k}\right) \quad \text { for }\left|L-L_{k}\right|<\frac{\Delta L}{2} \\
& D e\left(L_{i}-L_{k}\right) \quad\left\{\begin{array}{c}
1 \text { if }\left|L_{i}-L_{k}\right| \leq \frac{\Delta L}{2} \\
0 \text { others }
\end{array}\right. \\
& T h\left[L_{k}, L_{\min }\left(d_{i}\right), L_{\max }\left(d_{i}\right)\right]=\left\{\begin{array}{c}
\text { if }\left\{\begin{array}{c}
L_{k}<L_{\min }\left(d_{i}\right)-\frac{\Delta L}{2} \\
0
\end{array}\right. \\
\frac{L_{k}-L_{\min }\left(d_{i}\right)}{\Delta L}+\frac{1}{2} \text { if } \mid L_{k}-L_{\min }\left(d_{i}\right)+\frac{\Delta L}{2} \\
\frac{L_{k}-L_{\max }\left(d_{i}\right)}{\Delta L}+\frac{1}{2}
\end{array}\right. \\
& \text { if }\left|L_{k}-L_{\max }\left(d_{i}\right)\right| \leq \frac{\Delta L}{2} \\
& \text { others }
\end{aligned}
$$

Here $N$ is the total number of galaxies in the magnitude-limited sample set, and $N_{\mathrm{p}}$ is the total number of bins with bin width $\Delta L$. The index $k$ runs from $1,2,3, \ldots \ldots$ to $N_{\mathrm{p}}$. Here the step function $T h\left[L_{k}, L_{\min }\left(d_{i}\right), L_{\max }\left(d_{i}\right)\right]$, is different from that in EEP, because the upper limit of the integral in equation (1) is $L_{\max }\left(d_{i}\right)$ rather than infinity. We simply use the results obtained in the previous subsection, put them in equation (3) to compare the values (along with errors) for the discrete points derived in the second method to the Schechter function form (Figure 2). We have to take some care to normalize the values of $\phi_{k}^{\prime}$, if we want to compare these two maximum likelihood fits. The uncertainty of each dot is obtained using equation (3), using straightforward error propagation (Bevington \& Robinson 1992).

We can see from the figure that these two methods are consistent with each other for the Schechter parameters from the previous subsection, implying that the Schechter function is a good fit to the data. This remained true for other choices of bin widths, $\Delta L / L^{*}=1$ and $\Delta L / L^{*}=0.1$ as well. 


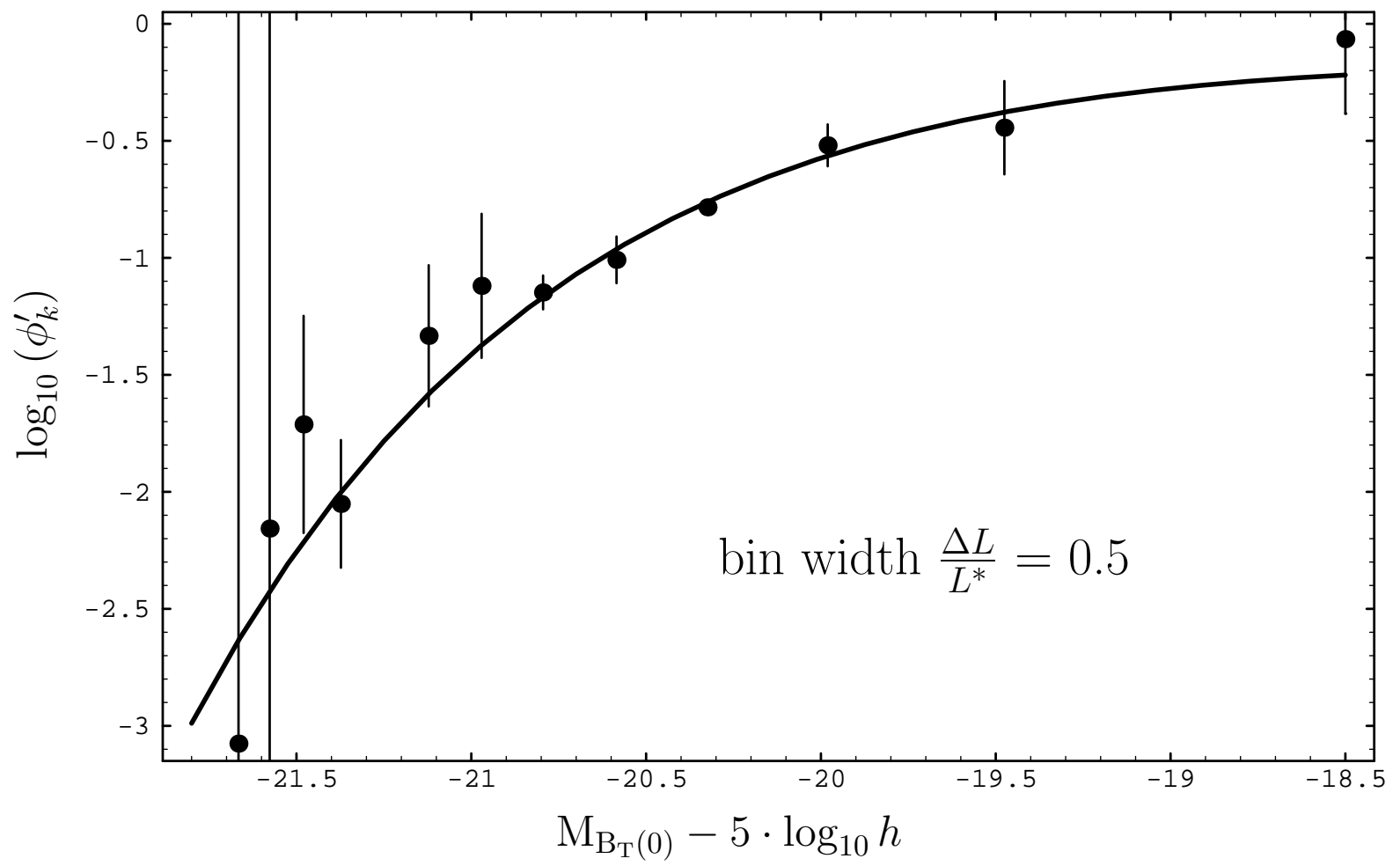

Fig. 2. Comparison between the step-wise maximum likelihood method (discrete dots) and the STY method (solid curve). We use results from Section 2.1, and bin width $\Delta L / L^{*}=0.5$ for the step-wise method in this plot.

\subsection{The normalization constant}

We calculate the normalization constant $\phi^{*}$ using standard techniques (EEP or Loveday et al. (1992)). This involves calculating the average space density of galaxies in the luminosity range being probed, $\bar{n}$, as follows:

$$
\bar{n}=\frac{\sum_{i=1}^{N} w\left(d_{i}\right)}{\int_{d_{\min }}^{d_{\max }} d x 4 \pi x^{2} S(x) w(x)}
$$

where

$$
\begin{aligned}
S\left(d_{i}\right) & =\frac{\int_{\max \left(L_{\min }\left(d_{i}\right), L_{1}\right)}^{\min \left(L_{\max }\left(d_{i}\right)\right.} \phi(L) d L}{\int_{L_{1}}^{L_{2}} \phi(L) d L} \\
w\left(d_{i}\right) & =\frac{1}{1+4 \pi \bar{n} J_{3}\left(r_{\mathrm{c}}\right) S\left(d_{i}\right)}, \quad J_{3}\left(r_{\mathrm{c}}\right)=\int_{0}^{r_{\mathrm{c}}} r^{2} \xi(r) d r
\end{aligned}
$$

$N$ is the total number of galaxies in the sample set, $d_{i}$ is the distance of each galaxy, and $d_{\min }$ and $d_{\max }$ are the nearest and the furthest distances among galaxies in the sample set. We have 
$d_{\min }=6.95 h^{-1} \mathrm{Mpc}$, and $d_{\max }=57.79 h^{-1} \mathrm{Mpc} . L_{1}$ and $L_{2}$ are related to the selection function $S\left(d_{i}\right)$ and the weighting function $w\left(d_{i}\right)$, but these can, with sufficient accuracy for our purposes be chosen to be $L_{\min }\left(d_{i}\right)$ and $L_{\max }\left(d_{i}\right)$, the dimmest and the brightest luminosities from our sample set. Finally, $\xi(r)$ is the two-point galaxy correlation function with $r_{\mathrm{c}} \approx 20 h^{-1}$ Mpc, and we use $4 \pi J_{3} \approx 10,000 h^{-3} \mathrm{Mpc}^{3}$ here (Efstathiou et al. 1988, Loveday et al. 1992).

After applying the Schechter function in the selection function $S\left(d_{i}\right)$, we find $\bar{n}=9.65 \times$ $10^{-4} h^{3} \mathrm{Mpc}^{-3}$ from equation (4). The normalization constant is equal to

$$
\phi^{*}=\frac{\bar{n}}{\int_{L_{1}}^{L_{2}} \phi(L) d L}
$$

So we get $\phi^{*}=1.34 \times 10^{-3} h^{3} \mathrm{Mpc}^{-3}$. The uncertainty of $\bar{n}$ is approximated by (Loveday et al. 1992)

$$
\delta \bar{n} \approx \sqrt{\frac{\bar{n}}{\int_{d_{\min }}^{d_{\max }} w S d V}}
$$

We thus find $\delta \bar{n} / \bar{n} \approx 0.19$. This value is larger than that in Loveday et al. (1992), because $\delta \bar{n} / \bar{n}$ is inversely proportional to the square root of number of galaxies in the sample set. There are 131 galaxies in our sample set, but there were about 1600 galaxies in Loveday et al. (1992). We thus find the uncertainty of $\phi^{*}$ by standard methods of error propagation, and hence obtain our final result, $\phi^{*}=(1.34 \pm 0.30) \times 10^{-3} h^{3} \mathrm{Mpc}^{-3}$.

\section{MODELS OF VELOCITY DISPERSION}

We have already mentioned the importance of using a self-consistent analysis of dark matter velocity dispersions when attempting to model galaxies as lenses (Cheng \& Krauss 2000). Here we describe such an analysis for various galaxy models. The key point is that the determination of $\sigma_{\mathrm{DM}}$ is a sensitive function of the galaxy anisotropy parameters and core radius, and one must consider this sensitivity when determining $\sigma_{\mathrm{DM}}$ on the basis of various observations. A theoretical dynamical analysis suggests that measuring line of sight velocity dispersions at the de Vaucouleurs effective radius $R_{\mathrm{e}}$ (1948), can provide a relatively robust estimate of the underlying $\sigma_{\mathrm{DM}}$, and also allow one to extract out contributions due to central mass concentrations in the galaxy.

Throughout our analysis we model the mass density distribution of elliptical galaxies with the following form (Hinshaw \& Krauss 1987):

$$
\rho=\frac{\sigma_{\mathrm{DM}}^{2}}{2 \pi G\left(r^{2}+r_{\mathrm{c}}^{2}\right)}
$$

where $\sigma_{\mathrm{DM}}$ (independent of radius $r$ ) is the velocity dispersion of this system (presumably 

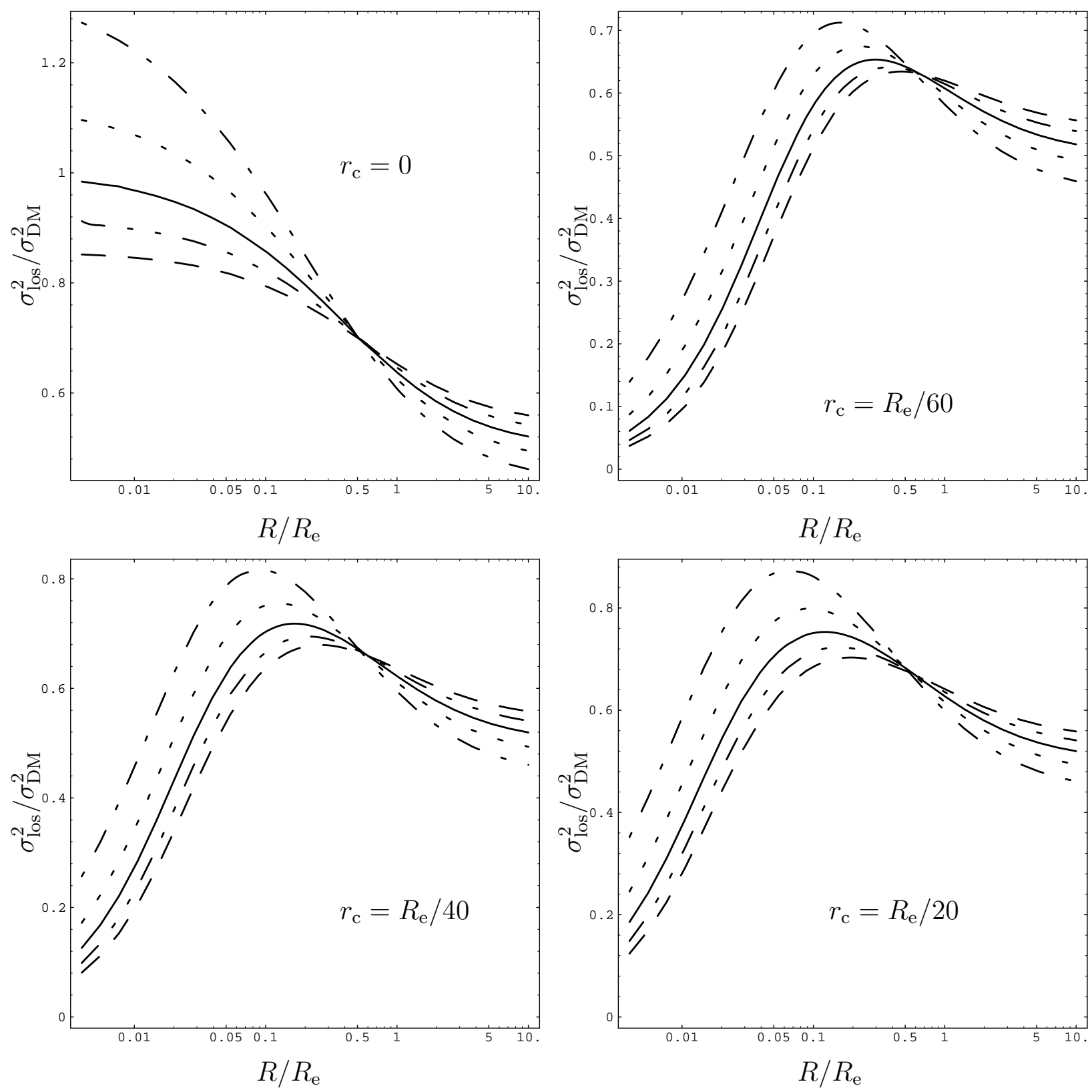

Fig. 3. Jaffe (1983) luminosity profile with core radii, $0, R_{\mathrm{e}} / 20, R_{\mathrm{e}} / 40$, and $R_{\mathrm{e}} / 60$. The five curves in each plot, dash-dot curve, dot-dot curve, solid curve, dash-dot-dot curve, and dash-dash curve, represent the five different anisotropy parameters $\beta=0.4,0.2,0.0,-0.2$, and -0.4 , respectively.

the dominant dark matter), and $r_{\mathrm{c}}$ is the core radius. When $r_{\mathrm{c}}$ is zero, this model reverts to a singular isothermal sphere (SIS) model. We should bear in mind that the velocity dispersion in this equation, which is assumed to be independent of radius, cannot be measured directly. What we can measure is the line-of-sight velocity dispersion $\sigma_{\text {los }}(R)$, with the projected distance $R$ measured from the center of the observed galaxy (i.e., $R$ is perpendicular to the lineof-sight to us). This means that we need to integrate over the line-of-sight distance in order to work backward to infer the value of $\sigma_{\mathrm{DM}}$ for theoretical purposes. For a singular isothermal sphere, $\sigma_{\mathrm{DM}}$ is not a function of $R$, but $\sigma_{\text {los }}(R)$ is, and this relationship is different when a 

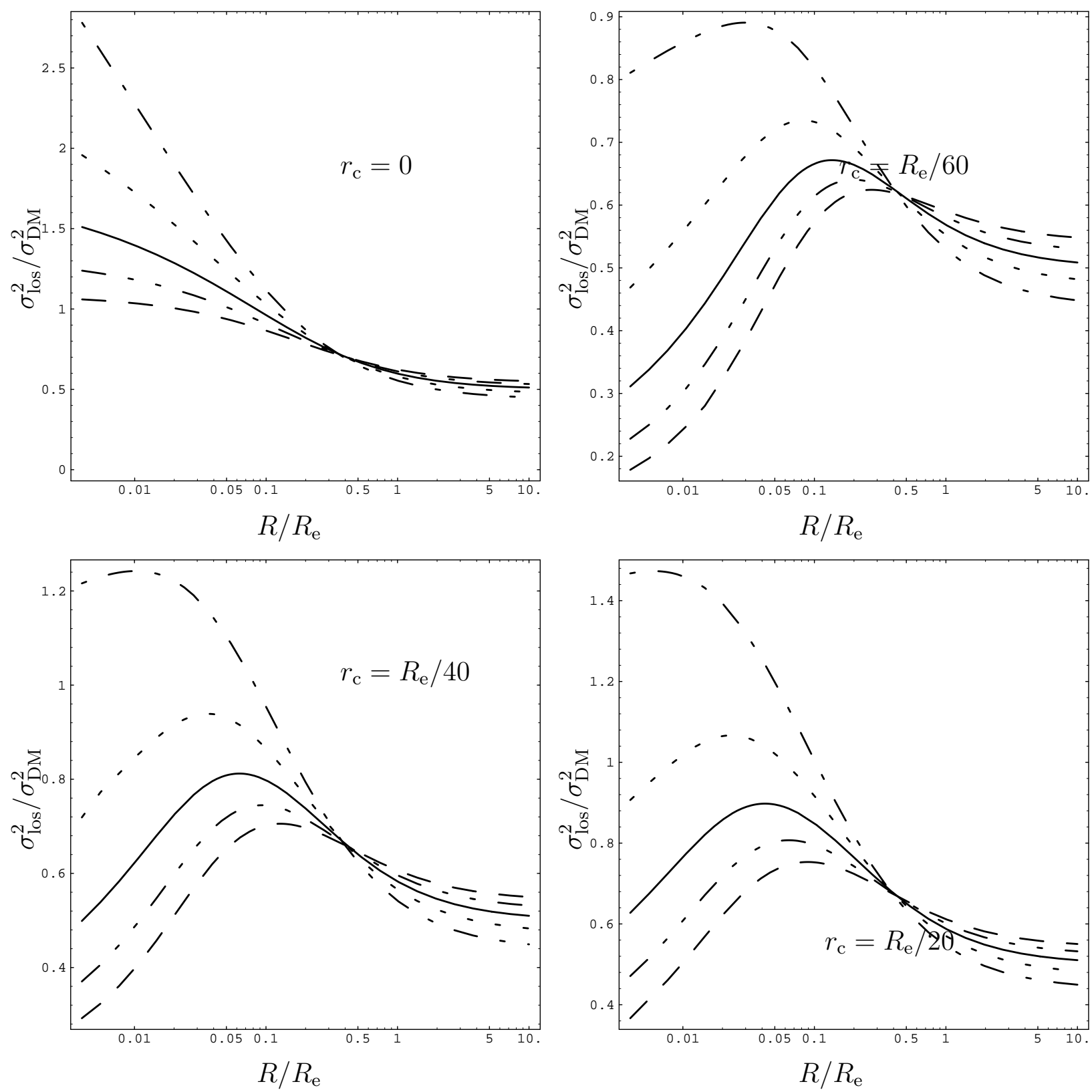

Fig. 4. Hernquist (1990) luminosity profile with core radii, $0, R_{\mathrm{e}} / 20, R_{\mathrm{e}} / 40$, and $R_{\mathrm{e}} / 60$. The description of the five curves in each plot is the same as in Figure 3.

galaxy has a finite core (although this is a secondary issue). Thus, one has to be careful how to use the measured velocity dispersion to derive the relevant quantity to utilize for lensing, namely whether it well approximates $\sigma_{\mathrm{DM}}$. To find the relationship between $\sigma_{\mathrm{DM}}$ and $\sigma_{\mathrm{los}}(R)$, we start from Jeans equations as follows (Ogorodnikov 1965,Binney \& Tremaine 1987):

$$
\frac{d}{d r}\left(\nu \overline{\sigma_{\mathrm{r}}^{2}}\right)+2 \beta \frac{\nu \overline{\sigma_{\mathrm{r}}^{2}}}{r}=-\nu \frac{d \Phi}{d r}
$$


where

$$
\frac{\partial \Phi}{\partial r}=\frac{4 \pi G}{r^{2}} \int_{0}^{r} d r^{\prime} \cdot r^{\prime 2} \rho\left(r^{\prime}\right)=2 \sigma_{\mathrm{DM}}^{2}\left[\frac{1}{r}-\frac{r_{\mathrm{c}}}{r^{2}} \arctan \left(\frac{r}{r_{\mathrm{c}}}\right)\right]
$$

by using equation (7).

In equation $(8), \nu(r)$ is the luminosity profile, $\sigma_{\mathrm{r}}(r)$ is the radial part of the velocity dispersion of the observed galaxy at distance $r$ from the center of that galaxy. The other two components of the velocity dispersion $\sigma$ in spherical coordinates are $\sigma_{\theta}$ and $\sigma_{\phi}$. We define the anisotropy $\beta(r)$ as $\overline{\sigma_{\theta}^{2}}=\overline{\sigma_{\phi}^{2}}=(1-\beta) \overline{\sigma_{\mathrm{r}}^{2}}$.

The line-of-sight velocity dispersion $\sigma_{\text {los }}(R)$ is related to $\nu \overline{\sigma_{\mathrm{r}}^{2}}$ by

$$
I(R) \sigma_{\mathrm{los}}^{2}(R)=2 \int_{R}^{\infty}\left(1-\beta \frac{R^{2}}{r^{2}}\right) \nu \overline{\sigma_{\mathrm{r}}^{2}} \frac{r}{\sqrt{r^{2}-R^{2}}} d r
$$

where $I(R)$ is the surface brightness profile, which can be calculated as follows:

$$
I(R)=2 \int_{R}^{\infty} \frac{\nu r}{\sqrt{r^{2}-R^{2}}} d r
$$

By solving the differential equation (8), we cam re-write equation (10) as

$$
I(R) \sigma_{\operatorname{los}}^{2}(R)=2 \int_{0}^{\infty} d u\left(1-\beta \frac{R^{2}}{R^{2}+u^{2}}\right) \frac{1}{\left(R^{2}+u^{2}\right)^{\beta}} \int_{\sqrt{R^{2}+u^{2}}}^{\infty} d x \cdot x^{2 \beta} \nu(x) \frac{\partial \Phi}{\partial x}
$$

If we assume $\beta$ is simply a constant independent of $r$ in equations (9), (11), and (12), then once we know the form for the luminosity profile $\nu(r)$, we know the ratio of $\sigma_{\text {los }}^{2} / \sigma_{\text {DM }}^{2}$ at the different projected distance $R$. We have considered two different but similar luminosity profiles $\nu(r)$. We adopt $\nu(r) \propto r^{-2}\left(r+r_{0}\right)^{-2}$, with $r_{0}=1.31 R_{\mathrm{e}}$, from Jaffe (1983), and $\nu(r) \propto r^{-1}\left(r+r_{0}\right)^{-3}$, with $r_{0}=0.45 R_{\mathrm{e}}$, from Hernquist (1990). We performed our numerical calculations by adjusting $\beta=0.4,0.2,0,-0.2,-0.4$ and $r_{\mathrm{c}}=0, \frac{1}{20} R_{\mathrm{e}}, \frac{1}{40} R_{\mathrm{e}}, \frac{1}{60} R_{\mathrm{e}}$ for both Jaffe (1983) and Hernquist (1990) luminosity profiles (Figure 3 and Figure 4). The ratio of the core radius to the effective radius may be estimated from Lauer (1985), and we find that this ratio is between $1 / 20$ and $1 / 60$ for class I galaxies in Lauer (1985). From Figure 3 and Figure 4 , we clearly see that $\sigma_{\text {los }}^{2} / \sigma_{\mathrm{DM}}^{2}$ is strongly sensitive to the anisotropy when the projected radius is less than $0.1 R_{\mathrm{e}}$ (whether or not the core radius is non-zero, a factor to which the result is also sensitive). This is consistent with observational data (Bender, Saglia \& Gerhard 1994). It can thus be dangerous to simply consider the line-ofsight velocity dispersion within $0.1 R_{\mathrm{e}}$ as $\sigma_{\mathrm{DM}}$. Even if the core radius is effectively very small, 
as numerous observations of ellipticals suggest, the sensitivity to the anisotropy parameter implies one should be careful when interpreting meassured values of the velocity dispersion.

However, if we are careful to look at all the 40 curves in Figure 3 and Figure 4, we can find the following inequalities:

$$
\begin{array}{ll}
1.16 \leq \frac{\sigma_{\mathrm{DM}}}{\sigma_{\operatorname{los}}(R)} \leq 1.27 & \text { at } R=0.4 R_{\mathrm{e}} \\
1.20 \leq \frac{\sigma_{\mathrm{DM}}}{\sigma_{\operatorname{los}}(R)} \leq 1.30 & \text { at } R=0.54 R_{\mathrm{e}} \\
1.24 \leq \frac{\sigma_{\mathrm{DM}}}{\sigma_{\operatorname{los}}(R)} \leq 1.37 & \text { at } R=R_{\mathrm{e}}
\end{array}
$$

These indicate that the intrinsic scatter of $\sigma_{\mathrm{DM}}$ will be less than 10 per cent if we can measure the line-of-sight velocity dispersion at $R_{\mathrm{e}}$ or half $R_{\mathrm{e}}$. Then, we can multiply this velocity dispersion by the average value (e.g., 1.31 for $R=R_{\mathrm{e}}$, or 1.25 for $R=0.54 R_{\mathrm{e}}$ ), in order to get $\sigma_{\mathrm{DM}}$. We can see that this argument is almost independent of the core radius of each galaxy and the anisotropy parameter $\beta$. We consider below the velocity dispersions at two different $R$ values as a consistency check of our analysis. First however, we comment on another important feature of these figures which is relevant to a determination of galaxy parameters for lensing purposes.

Kochanek (1996) has pointed out that the inclusion of a core radius will in general require, for self-consistency, a larger value of $\sigma_{\mathrm{DM}}$ to be used in models, and has argued that this will cancel out the effect that non-zero core radii can have on suppressing lensing optical depths if the value of $\sigma_{\mathrm{DM}}$ were instead kept fixed. Examining Figures 3 and 4 at approximately 0.5 $R_{\mathrm{e}}$, where the different curves intersect, indeed illustrates the effect described by Kochanek. As the core radius is increased $\sigma_{\mathrm{DM}}$ increases. However the key point is that the change in $\sigma_{\mathrm{DM}}$ is small compared to the huge variances in $\sigma_{\mathrm{DM}}$ one sees in models with different values of $\beta$, and also is small compared to the possibility of overestimating $\sigma_{\mathrm{DM}}$ if central line-of-sight velocity dispersions are used.

Note that in this section we chose 42 galaxies from 12 different data sets to be our galaxy sample, because for these we could find or estimate the velocity dispersions at both $R_{\mathrm{e}}$ and $0.54 R_{\mathrm{e}}$. Among those 12 sets of observations, most use a slit size from one to three arcseconds. This should therefore not be significant when $R_{\mathrm{e}}$ is on the order of ten arcseconds, as it is in this paper (see Table 1). Furthermore, from Figures 3 and 4, one can see that the velocity curves are relatively smooth between $R_{\mathrm{e}}$ and $0.54 R_{\mathrm{e}}$ (i.e., theoretically, $\sigma_{\mathrm{DM}}=1.31 \sigma_{R_{\mathrm{e}}}=$ $\left.1.25 \sigma_{0.54 R_{\mathrm{e}}}\right)$. This indicates that even if the slit size were up to half $R_{\mathrm{e}}$, this effect might still not strongly bias the results.

\subsection{Velocity dispersions at $R=R_{\mathrm{e}}$}

Based on values of $R_{\mathrm{e}}$ from RC3, we can estimate velocity dispersions $\sigma_{\mathrm{DM}}$ of these 42 galaxies. We also consider $R_{\mathrm{e}}$ from various different papers and translate the different values 
of velocity dispersions into the uncertainty of the velocity dispersion measured at $R_{\mathrm{e}}$, as given by RC3. We would quote either this uncertainty or the uncertainty from the observations, whichever is larger. The other problem is that different observers might use different methods to measure velocity dispersions. We calculate the weighted mean of all these velocity dispersions for one galaxy as our final line-of-sight velocity dispersion of that galaxy (Lauer 1985,Bevington \& Robinson 1992). We list the information of $R_{\mathrm{e}}, \mathrm{B}_{\mathrm{T}}(0)$, distances and velocity dispersions of all 42 galaxies in Table 1.

Using Table 1 and a suitable value for $\mathrm{M}_{\mathrm{B}_{\mathrm{T}}(0)}^{*}=-19.66+5 \log _{10} h \pm 0.30$ as discussed in Section 2, we can calculate the least-square-fit of the relation between $\log _{10}\left(L / L^{*}\right)$ and $\log _{10}\left(\sigma_{R_{\mathrm{e}}} / \mathrm{km} \mathrm{s}^{-1}\right)$ (Figure 5$)$. We exclude galaxies NGC 4251, NGC 7796, NGC 4486B, and NGC 6411, because the former two galaxies do not have available distances from $7 \mathrm{~s}$, and the latter two galaxies are extreme outlyers (One galaxy is the farthest right one and the other galaxy is the lowest one in Figure 5.), and there is no available observational value of $R_{\mathrm{e}}$ of NGC 4486B in RC3. We obtain

$$
\log _{10}\left(L / L^{*}\right)=(-4.04 \pm 0.49)+(1.89 \pm 0.22) \log _{10}\left(\sigma_{R_{\mathrm{e}}} / \mathrm{km} \mathrm{s}^{-1}\right)
$$

with $\chi^{2}=48$ for 38 galaxies in Figure 5. We use both uncertainties of luminosities and velocity dispersions to work out uncertainties in equation (13) and its $\chi^{2}$. If we set $L=L^{*}$ in equation (13), then we find the velocity dispersion of luminous elliptical galaxies at the effective radius, $\sigma_{R_{\mathrm{e}}}^{*}=136 \pm 15 \mathrm{~km} \mathrm{~s}^{-1}$. From the discussion in Section 3, we then multiply this number by 1.31 , to get $\sigma_{\mathrm{DM}}^{*} \approx 1.31 \sigma_{R_{\mathrm{e}}}^{*} \approx 178 \mathrm{~km} \mathrm{~s}^{-1}$.

In order to consider the confidence levels of the velocity dispersion we determine here, we set $\Delta \chi^{2}=3.5$ for the 68 per cent confidence level of velocity dispersion, and 7.8 for the 95 per cent confidence level (Lampton, Margon \& Bowyer 1976), because we actually have three adjustable parameters $\left(\sigma_{R_{\mathrm{e}}}^{*}, L^{*}\right.$, and the power law between $\sigma_{R_{\mathrm{e}}}$ and $\left.L / L^{*}\right)$, even though we only vary $\sigma_{R_{\mathrm{e}}}^{*}$ and fix the other two parameters. The final 95 per cent confidence level is then determined to be $\sigma_{\mathrm{DM}}^{*}=178_{-28}^{+29} \mathrm{~km} \mathrm{~s}^{-1}$.

Several issues are relevant to this result. In the first place, note that the $L$ vs $\sigma$ relation in the above equation differs from the standard Faber-Jackson relation (Faber \& Jackson 1976). Note however that this FJ relation is appropriate for central velocity dispersions. In addition, it may be there case that the elliptical galaxies do not form a uniform population, but rather that bright galaxies and faint galaxies follow separate Faber-Jackson curves (this point was raised to us by J. Peebles, who has been investigating this issue (Fukugita \& Peebles 1999)). Nakamura and Suto (1997) have also pointed out how the value of the power law relation can differ from 4. It thus may not be appropriate to enforce this relation on the bulk sample. To explore this latter possibility, and because it is galaxies with the largest velocity dispersions which will dominate in the analysis of lensing statistics, we considered a subset of our galaxy sample with $\log _{10}\left(\sigma_{R_{\mathrm{e}}} / \mathrm{km} \mathrm{s}^{-1}\right)>2.2$ (i.e., 23 galaxies) and rederived the $L$ - $\sigma$ relation. In this case, we find:

$$
\log _{10}\left(L / L^{*}\right)=(-6.20 \pm 1.82)+(2.83 \pm 0.79) \log _{10}\left(\sigma_{R_{\mathrm{e}}} / \mathrm{km} \mathrm{s}^{-1}\right)
$$




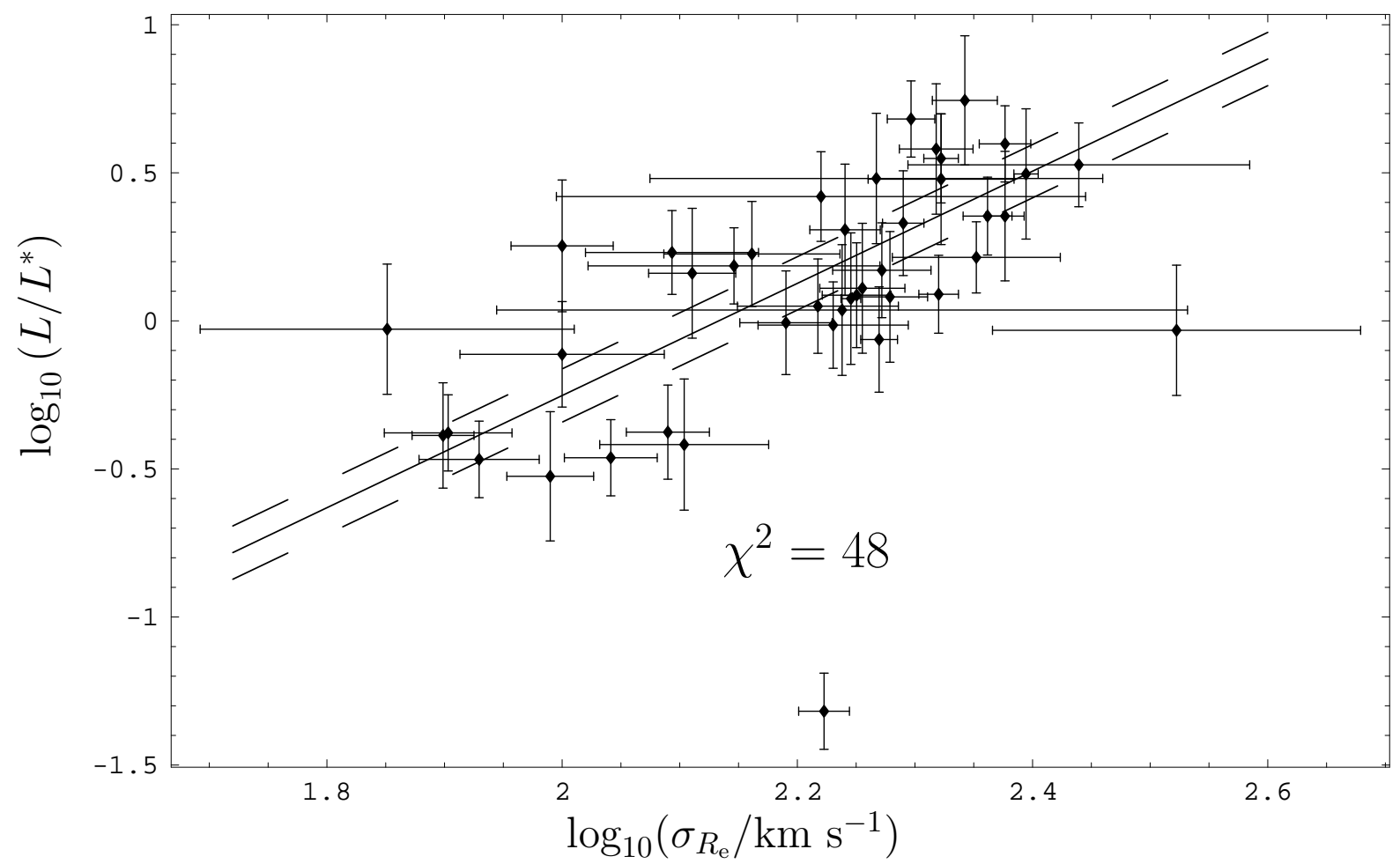

Fig. 5. Least-square-fit of $\log _{10}\left(L / L^{*}\right)$ vs. $\log _{10}\left(\sigma_{R_{\mathrm{e}}} / \mathrm{km} \mathrm{s}^{-1}\right)$. The center solid line is the best fit, as shown in equation (13). The upper dash line is the best fit plus 0.09 , and the lower dash line is the best fit minus 0.09 . These two lines are the 95 per cent confidence limits.

with $\chi^{2}=23.6$ and $\sigma_{R_{\mathrm{e}}}^{*}=155 \mathrm{~km} \mathrm{~s}^{-1}$. In this case, we find a somewhat higher value of $\sigma_{\mathrm{DM}}^{*}=203 \mathrm{~km} \mathrm{~s}^{-1}$ as might be expected given the features of this subsample.

A recent result from galaxy-galaxy weak lensing (Fischer et al. 2000) constrains the velocity dispersion $\sigma_{v}$ of the average foreground galaxy to be $150-190 \mathrm{~km} \mathrm{~s}^{-1}$ and this result translates to a $\sigma_{\mathrm{DM}}$ (using our notation) of the average foreground galaxy to be $210-266 \mathrm{~km} \mathrm{~s}^{-1}$. It is interesting to compare this result with the estimate we would derive here. Based on the luminosity of the average foreground galaxy given in Fischer et al. (2000), i.e., $8.7 \times 10^{9} L_{\text {sun }}$ in the g' band, our formula would predict a $\sigma_{\mathrm{DM}}$ around $220 \mathrm{~km} \mathrm{~s}^{-1}$, which is in good agreement with the range of $\sigma_{\mathrm{DM}}$ determined by Fischer.

\subsection{Velocity dispersions at $R=0.54 R_{\mathrm{e}}$}

Another fit between luminosities and velocity dispersions of sample galaxies has been calculated at $R=0.54 R_{\mathrm{e}}$. Velocity dispersions at $R=0.54 R_{\mathrm{e}}$ are estimated and listed in Table 1 . We still exclude NGC 4251, NGC 7796, and NGC 4486B, for the same reasons described in the above section. We find

$$
\log _{10}\left(L / L^{*}\right)=(-5.01 \pm 0.55)+(2.28 \pm 0.24) \log _{10}\left(\sigma_{0.54 R_{\mathrm{e}}} / \mathrm{km} \mathrm{s}^{-1}\right)
$$




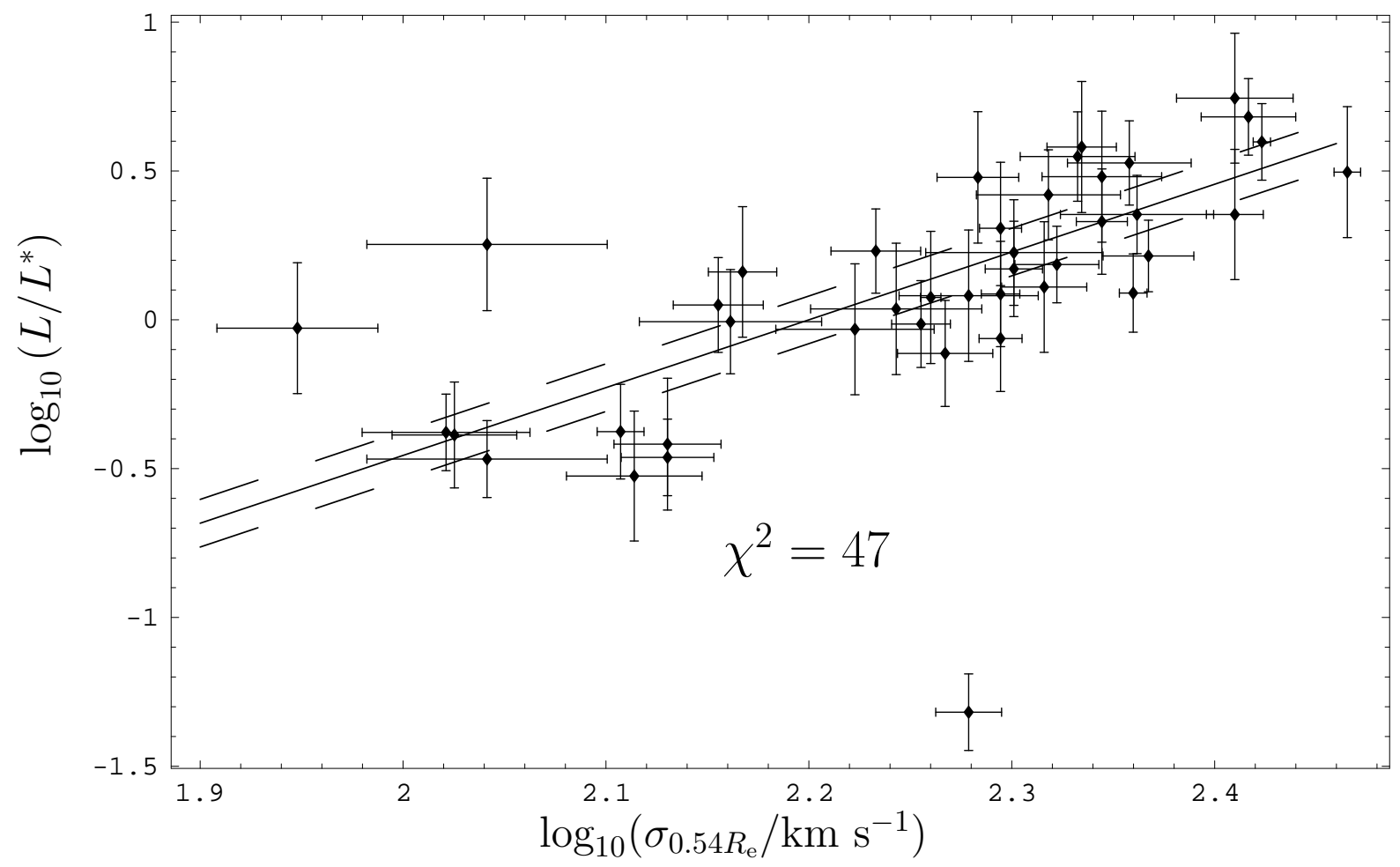

Fig. 6. Least-square-fit of $\log _{10}\left(L / L^{*}\right)$ vs. $\log _{10}\left(\sigma_{0.54 R_{\mathrm{e}}} / \mathrm{km} \mathrm{s}^{-1}\right)$. The solid line is the best fit, as shown in equation (15). The upper dash line is the best fit plus 0.08, and the lower dash line is the best fit minus 0.08 . These two lines are the 95 per cent confidence limits.

with $\chi^{2}=47$ and $\sigma_{0.54 R_{\mathrm{e}}}^{*}=159 \pm 15 \mathrm{~km} \mathrm{~s}^{-1}$ (Figure 6). As we have discussed in Section 3, if we multiply this number by 1.25 , then we should get the expected $\sigma_{\mathrm{DM}}^{*}$. Therefore, $\sigma_{\mathrm{DM}}^{*} \approx$ $1.25 \sigma_{0.54 R_{\mathrm{e}}}^{*}=198 \mathrm{~km} \mathrm{~s}^{-1}$ in this case. If we consider a subset of our galaxy sample with $\log _{10}\left(\sigma_{0.54 R_{\mathrm{e}}} / \mathrm{km} \mathrm{s}^{-1}\right)>2.2$ (i.e., 28 galaxies), we find

$$
\log _{10}\left(L / L^{*}\right)=(-6.43 \pm 1.34)+(2.88 \pm 0.58) \log _{10}\left(\sigma_{0.54 R_{\mathrm{e}}} / \mathrm{km} \mathrm{s}^{-1}\right)
$$

with $\chi^{2}=23$ and $\sigma_{0.54 R_{\mathrm{e}}}^{*}=169 \mathrm{~km} \mathrm{~s}^{-1}$. In this case, we find $\sigma_{\mathrm{DM}}^{*}=211 \mathrm{~km} \mathrm{~s}^{-1}$.

We see that the two slopes in $L-\sigma$ relation obtained at different values of $R_{\mathrm{e}}$ are close to each other. Furthermore, the inferred value of $\sigma_{\mathrm{DM}}^{*}$ at $R=0.54 R_{\mathrm{e}}$ is somewhat larger than the value of $\sigma_{\mathrm{DM}}^{*}$ at $R=R_{\mathrm{e}}$. This can be explained as follows. The above estimates are for the case of a purely finite isothermal distribution. If one adds to this distribution some central mass, such as a large central black hole, this will further increase the central velocity dispersion, and also change the relationship between $\sigma_{\mathrm{DM}}$ and the line-of-sight velocity dispersion at $R_{\mathrm{e}}$ and half $R_{\mathrm{e}}$. By measuring the velocity dispersions at both points, however, one can hope to extract out the central mass contribution and also the isothermal contribution. (Alternately, it is clear that the velocity dispersion at $R_{\mathrm{e}}$ will be less sensitive to the former contribution, and thus can be used to approximate the isothermal contribution.) It is important to recognize that the central mass contribution can affect the velocity dispersion more significantly than it affects the optical depth for lensing, so that incorporating this contribution in an effective isothermal sphere velocity dispersion will lead to an overestimate 
of the optical depth.

As a quick demonstration, let us see how we can extract out the central mass contribution. If we simply assume $\nu(x) \propto 1 / x^{4}$ due to a point mass at the center of galaxy, from equations (11) and (12), we can obtain

$$
\sigma_{\text {los }}^{2}(R)=\frac{G M}{6 R}
$$

where $M$ is the mass of that point mass. Then, we can set up equations as follows:

$$
\begin{aligned}
\sigma_{\text {los }}^{2}\left(R_{\mathrm{e}}\right) & =\sigma_{\text {los,g }}^{2}\left(R_{\mathrm{e}}\right)+\frac{G M}{6 R_{\mathrm{e}}} \\
\sigma_{\mathrm{los}}^{2}\left(0.54 R_{\mathrm{e}}\right) & =\sigma_{\mathrm{los}, \mathrm{g}}^{2}\left(0.54 R_{\mathrm{e}}\right)+\frac{G M}{6 \cdot\left(0.54 R_{\mathrm{e}}\right)}
\end{aligned}
$$

where $\sigma_{\text {los,g }}(R)$ is the isothermal contribution to the velocity dispersion of that galaxy as a function of $R$. As discussed above, we know that $1.31 \sigma_{\mathrm{los}, \mathrm{g}}\left(R_{\mathrm{e}}\right)=1.25 \sigma_{\mathrm{los}, \mathrm{g}}\left(0.54 R_{\mathrm{e}}\right)=\sigma_{\mathrm{DM}}$. Thus, the appropriate isothermal effective $\sigma_{\mathrm{DM}}$ can be calculated for each galaxy and the value of $\sigma_{\mathrm{DM}}^{*}$ can be found through a least-square-fit method. A very quick estimation of the appropiate $\sigma_{\mathrm{DM}}^{*}$ from equations $(14)$ and (16), is $\sigma_{\mathrm{DM}}^{*} \approx 190 \mathrm{~km} \mathrm{~s}^{-1}$, with the slope of the $L$ vs. $\sigma_{\mathrm{DM}}$ relation being $\approx 2.8$. It is interesting to note that when values close to these are utilized in an analysis of lensing statistics (Cheng \& Krauss 2000) one obtains mean angular splittings that are comparable to the observed splittings.

\section{DISCUSSIONS}

First note that both $\mathrm{M}_{\mathrm{B}_{\mathrm{T}}(0)}^{*}$ and $\alpha$, fitted to the Schechter function are close to the results in Loveday et al. (1992). This is expected, because the $\mathrm{B}_{\mathrm{T}}$ and $\mathrm{B}_{\mathrm{J}}$ bands are close. In fact, we would expect our $\mathrm{M}_{\mathrm{B}_{\mathrm{T}}(0)}^{*}$ to be slightly fainter than $\mathrm{M}_{\mathrm{B}_{\mathrm{J}}}^{*}\left(=-19.71+5 \log _{10} h\right)$, because the $\mathrm{B}_{\mathrm{J}}$ band is measured at a longer wavelength and most galaxy luminosities are peaker toward the redder end of the spectrum. On the other hand, our $\mathrm{M}_{\mathrm{B}_{\mathrm{T}}(0)}^{*}$ is brighter than $\mathrm{M}_{\mathrm{B}_{\mathrm{T}}}^{*}(=$ $\left.-19.37+5 \log _{10} h\right)$ given in EEP. Given the Galactic extinction correction, this is also what we would expect. We have also shown that the Schechter $\alpha$ of elliptical galaxies is shallower than that for total galaxies, which is consistent with EEP and Loveday et al. (1992).

The ratio of our $\phi^{*}$ to the one in Loveday et al. (1992) is about 9.6 per cent. In comparison with the $\phi^{*}$ in Mobasher, Sharples \& Ellis (1993), the ratio is about 12 per cent. These two numbers indicate the fraction of elliptical galaxies out of total galaxies, and they are consistent with the conclusion of Postman \& Geller (1984), of $12 \pm 2$ per cent for this ratio. Our results here suggest that the population of elliptical galaxies is $(10.8 \pm 1.2)$ per cent of all galaxies. 
Regarding our luminosity function determination, it is of course still possible that the galaxies in our sample set do not form a complete sample set. Currently, we calculate $\left\langle\frac{\mathrm{V}}{\mathrm{V}_{\max }}\right\rangle=$ 0.45 (Schmidt 1968). If we had an incomplete elliptical galaxy sample set, and if we would believe those 'missing' E galaxies are near apparent magnitude 12.19, then we would expect $<\frac{\mathrm{V}}{\mathrm{V}_{\max }}>$ larger than 0.45 . Also, according to the theoretical prediction in Zucca, Pozzetti \& Zamorani (1994), we would then have an even a dimmer $\mathrm{M}^{*}$ and a steeper $\alpha$. A fainter $\mathrm{M}^{*}$ would result an even smaller $\sigma_{\mathrm{DM}}^{*}$ than the value calculated in Section 3.1 and Section 3.2.

The analysis in Section 3.1, suggests $\sigma_{R_{\mathrm{e}}}^{*}=136 \pm 15 \mathrm{~km} \mathrm{~s}^{-1}$. It implies that $\sigma_{\mathrm{DM}}^{*}=178 \pm$ $29 \mathrm{~km} \mathrm{~s}^{-1}$. This uncertainty is large, because the uncertainties from distances of galaxies in the sample set and from $\mathrm{M}_{\mathrm{B}_{\mathrm{T}}(0)}^{*}$ are large. (Also note that the best fit value if we account for possible central mass concentrations is $\sigma_{\mathrm{DM}}^{*} \approx 190 \mathrm{~km} \mathrm{~s}^{-1}$, which falls within the range included above.) We also notice that $\mathrm{M}_{\mathrm{B}_{\mathrm{T}}(0)}^{*}$ is a sensitively coupled to $\sigma_{\mathrm{DM}}^{*}$. For example, if we use $\mathrm{M}_{\mathrm{B}_{\mathrm{T}}(0)}^{*}=-19.9+5 \log _{10} h$ (Fukugita \& Turner 1991,Kochanek 1994), then we will increase $\sigma_{\mathrm{DM}}^{*}$ by $8 \%-12 \%$. On the other hand, if we use $\mathrm{M}_{\mathrm{B}_{\mathrm{T}}(0)}^{*}=-19.5+5 \log _{10} h$, then we will decrease $\sigma_{\mathrm{DM}}^{*}$ by $5 \%-8 \%$. One should be careful about this issue in any strong gravitational lensing analysis. The completeness of our galaxy sample discussed in Section 2.1 does not seem to lead to a significant uncertainty by comparison. For example, different numbers of galaxies included in our sample produce a variation of $4 \%-6 \%$ in $\sigma_{\mathrm{DM}}$, which is within the values estimated from the uncertainty of $\mathrm{M}^{*}$ itself. It might be interesting to consider the relation between $\log _{10}\left(L / L^{*}\right)$ and $\log _{10}\left(\sigma / \mathrm{km} \mathrm{s}^{-1}\right)$ in other bands (e.g. $\mathrm{V}$ or $\mathrm{R}$ band, if the observation is possible; or a radio survey), to see whether including more elliptical galaxies would reduce the intrinsic scatter of this power law relation. If so, then the improved value of $\sigma_{\mathrm{DM}}^{*}$ and the power law from that survey would be a great help in the study of gravitational lensing statistics. Based on our present result, the optical depth of gravitational lenses would be expected to be reduced by 43 per cent compared to the one in Kochanek (1993), if all other parameters are unchanged.

In this paper, we treat the anisotropy parameter $\beta$ as a constant. We expect that our conclusion will not change too much even if $\beta$ is a function of radial distance $r$, as long as $|\beta| \leq$ 0.4 (Kochanek 1994) (Figure 3 and Figure 4).

Finally, the theoretical modelling in Section 3, suggests that $\sigma_{\operatorname{los}}^{2} / \sigma_{\mathrm{DM}}^{2}$ is strongly dependent on models and parameters within $0.1 R_{\mathrm{e}}$ (Figure 3 and Figure 4 ). We also observe that the ratio $\sigma_{\text {los }}^{2} / \sigma_{\mathrm{DM}}^{2}$ converges at about $0.5 R_{\mathrm{e}}$ for all different parameters. This suggests that we should use observational values of $\sigma_{\mathrm{los}}^{2} / \sigma_{\mathrm{DM}}^{2}$ between $0.4 R_{\mathrm{e}}$ and $R_{\mathrm{e}}$, if we want to reduce the intrinsic scatter from theoretical modelling. We hope observers will consistently measure velocity dispersions of elliptical galaxies at both $R_{\mathrm{e}}$ and $0.54 R_{\mathrm{e}}$ in order to refine our results. As we have discussed in Section 3.2, one can extract extra velocity dispersions at $R_{\mathrm{e}}$ (and $0.54 R_{\mathrm{e}}$ ), and get an effective value of $\sigma_{\mathrm{DM}}$ for each galaxy. As data improves, we expect that the techniques described here will be useful in further constraining input parameters in strong gravitational lensing analysis. 


\section{ACKNOWLEDGMENTS}

We would like to thank Harold Corwin, Deepak Jain, Peter Kernan, Jon Loveday, Paul Mason, Heather Morrison, Jim Peebles, Keith Robinson, Paul Schechter and the anonymous referee for very helpful input. This research work has been partially supported by the Industrial Physics Group in the Physics Department at Case Western Reserve University and by a grant to the particle astrophysic group at CWRU from the DOE. This research has also made use of the NASA/IPAC Extragalactic Database (NED) which is operated by the Jet Propulsion Laboratory, California Institute of Technology, under contract with the National Aeronautics and Space Administration.

\section{References}

Bender, R. 1990, A\&A, 229, 441 (Bender)

Bender, R., Saglia, R.P., Gerhard, O.E. 1994, MNRAS, 269, 785 (BSG)

Bertin, G., Bertola, F., Buson, L.M., Danziger, I.J., Dejonghe, H., Sadler, E.M., Saglia, R.P., de Zeeuw, P.T., Zeilinger, W.W. 1994, A\&A, 292, 381 (BZ)

Bevington, P.R., Robinson, D.K. 1992, Data Reduction and Error Analysis for the Physical Sciences. McGraw-Hill, New York

Binney, J., Tremaine, S. 1987, Galactic Dynamics. Princeton University Press, Princeton

Binney, J.J., Davies, R.L., Illingworth, G.D. 1990, ApJ, 361, 78 (BDI)

Carollo, C.M., de Zeeuw, P.T., van der Marel, R.P., Danziger, I.J., Qian, E.E. 1995, ApJ, 441, L25 (CQ)

Cheng, Y.-C.N., Krauss, L.M. 2000, International Journal of Modern Physics A, 15, 697

Davies, R.L., Birkinshaw, M. 1988, ApJS, 68, 409 (DB)

de Vaucouleurs, G. 1948, Ann. d'Ap., 11, 247

de Vaucouleurs, G., de Vaucouleurs, A., Corwin, H.G. Jr., Buta, R.J., Paturel, G., Fouqué, P. 1991, Third Reference Catalogue of Bright Galaxies. Springer-Verlag, New York (RC3)

Efstathiou, G., Ellis, R.S., Peterson, B.A. 1988, MNRAS, 232, 431 (EEP)

Faber, S.M., Jackson, R.E. 1976, ApJ, 204, 668

Faber, S.M., Wegner, G., Burstein, D., Davies, R.L., Dressler, A., Lynden-Bell, D., Terlevich, R.J. 1989, ApJS, 69, $763(7 \mathrm{~s})$

Fischer, P., McKay, T.A., Sheldon, E., et al. 2000, AJ, 120, 1198

Franx, M., Illingworth, G., Heckman, T. 1989, ApJ, 344, 613 (FIH) 
Fried, J.W., Illingworth, G.D. 1994, AJ, 107, 992 (FI)

Fukugita, M., Turner, E.L. 1991, MNRAS, 253, 99 (FT)

Fukugita, M., Peebles, P.J.E. 1999, ApJL, 524, 31

Hernquist, L. 1990, ApJ, 356, 359

Hinshaw, G., Krauss, L.M., 1987, ApJ, 320, 468 (HK)

Jaffe, W., 1983, MNRAS, 202, 995

Jedrzejewski, R., Schechter, P.J. 1989, AJ, 98, 147 (JS)

Kochanek, C.S. 1993, ApJ, 419, 12

Kochanek, C.S. 1994, ApJ, 436, 56

Kochanek, C.S. 1996, ApJ, 466, 638

Lampton, M., Margon, B., Bowyer, S. 1976, ApJ, 208, 177

Lauer, T.R. 1985, ApJ, 292, 104

Loveday, J., Peterson, B.A., Efstathiou, G., Maddox, S.J. 1992, ApJ, 390, 338

Mobasher, B., Sharples, R.M., Ellis, R.S. 1993, MNRAS, 263, 560

Nakamura, T.T., Suto, Y. 1997, Prog. of Theo. Phys., 97, 49

Ogorodnikov, K.F. 1965, Dynamics of Stellar Systems. Pergamon Press, Oxford

Postman, M., Geller, M.J. 1984, ApJ, 281, 95

Saglia, R.P., Bertin, G., Stiavelli, M. 1992, ApJ, 384, 433 (SBS)

Saglia, R.P., Bertin, G., Bertola, F., Danziger, J., Dejonghe, H., Sadler, E.M., Stiavelli, M., de Zeeuw, P.T., Zeilinger, W.W. 1993, ApJ, 403, 567 (SZ)

Sandage, A., Tammann, G.A., Yahil, A. 1979, ApJ, 232, 352 (STY)

Schechter, P.L. 1976, ApJ, 203, 297

Schmidt, M. 1968, ApJ, 151, 393

Turner, E.L., Ostriker, J.P., Gott, J.R. 1984, ApJ, 284, 1 (TOG)

Winsall, M.L., Freeman, K.C. 1993, A\&A, 268, 443 (WF)

Zucca, E., Pozzetti, L., Zamorani, G. 1994, MNRAS, 269, 953 


\begin{tabular}{|c|c|c|c|c|c|c|}
\hline Name & $R_{\mathrm{e}}\left({ }^{\prime \prime}\right)$ & Source & $m\left(\mathrm{~B}_{\mathrm{T}}(0)\right)$ & Distance $\left(h^{-1} \mathrm{Mpc}\right)$ & $\sigma_{R_{\mathrm{e}}}\left(\mathrm{km} \mathrm{s}^{-1}\right)$ & $\sigma_{0.54 R_{\mathrm{e}}}\left(\mathrm{km} \mathrm{s}^{-1}\right)^{c}$ \\
\hline NGC 636 & 19.4 & FIH & 12.16 & $15.0 \pm 1.8$ & $123 \pm 10$ & $128 \pm 3.4$ \\
\hline NGC 720 & 36.1 & BDI & 11.13 & $20.5 \pm 4.4$ & $174 \pm 12$ & $197 \pm 4.7$ \\
\hline NGC 777 & 34.4 & JS & 12.24 & $44.0 \pm 3.8$ & $275 \pm 92$ & $228 \pm 16$ \\
\hline NGC 1052 & 33.7 & $\mathrm{BDI} / \mathrm{DB} / \mathrm{FI}$ & 11.33 & $17.2 \pm 3.7$ & $176 \pm 3.4$ & $182 \pm 2.1$ \\
\hline NGC 1399 & 40.7 & $\mathrm{SBS} / \mathrm{WF}$ & 10.22 & $14.22 \pm 0.88$ & $230 \pm 11$ & $230 \pm 20$ \\
\hline NGC 1400 & 29.3 & $\mathrm{BZ}$ & 11.87 & $19.9 \pm 1.9$ & $170 \pm 25$ & $180 \pm 6.0$ \\
\hline NGC 1404 & 23.8 & $\mathrm{FIH} / \mathrm{WF}$ & 10.88 & $14.22 \pm 0.88$ & $209 \pm 8.1$ & $229 \pm 3.6$ \\
\hline NGC 1700 & 18.5 & BSG/FIH & 12.00 & $31.4 \pm 4.7$ & $195 \pm 7.9$ & $221 \pm 6.4$ \\
\hline NGC 2434 & 31.4 & $\mathrm{CQ}$ & 11.61 & $19.7 \pm 4.2$ & $190 \pm 14$ & $190 \pm 15$ \\
\hline NGC 2513 & 32.9 & JS & 12.48 & $46.6 \pm 9.9$ & $185 \pm 82$ & $221 \pm 15$ \\
\hline NGC 2663 & 54.6 & CQ & 10.23 & $22.4 \pm 4.7$ & $220 \pm 14$ & $257 \pm 17$ \\
\hline NGC 2778 & 15.7 & JS & 13.21 & $36.3 \pm 7.7$ & $71 \pm 26$ & $88.7 \pm 8.1$ \\
\hline NGC 3115 & 32.1 & BSG & 9.75 & $10.2 \pm 2.2$ & $100 \pm 10$ & $110 \pm 15$ \\
\hline NGC 3193 & 26.7 & BSG & 11.73 & $24.6 \pm 3.7$ & $145 \pm 25$ & $200 \pm 20$ \\
\hline NGC 3377 & 34.4 & BSG/FI & 10.98 & $8.6 \pm 1.3$ & $79.2 \pm 4.8$ & $106 \pm 7.5$ \\
\hline NGC 3379 & 35.2 & DB/SBS & 10.17 & $8.6 \pm 1.3$ & $186 \pm 6.7$ & $197 \pm 4.8$ \\
\hline NGC 3557 & 30.0 & FI & 10.79 & $24.0 \pm 5.1$ & $208 \pm 15$ & $216 \pm 8.5$ \\
\hline NGC 3608 & 33.7 & JS & 11.71 & $19.9 \pm 2.4$ & $165 \pm 26$ & $143 \pm 7.3$ \\
\hline NGC 3610 & 15.4 & BSG & 11.58 & $21.3 \pm 4.5$ & $129 \pm 11$ & $147 \pm 5.7$ \\
\hline NGC 3706 & 20.3 & $\mathrm{CQ}$ & 11.87 & $30.4 \pm 6.4$ & $238 \pm 8.9$ & $257 \pm 8.3$ \\
\hline NGC 4251 & 17.7 & BSG & 11.54 & $(10.14 \pm 0.56)^{b}$ & $85 \pm 10$ & $110 \pm 15$ \\
\hline NGC 4261 & 36.1 & DB & 11.32 & $27.8 \pm 5.9$ & $248 \pm 5.9$ & $292 \pm 4.4$ \\
\hline NGC 4278 & 34.4 & DB & 10.96 & $14.7 \pm 2.2$ & $178 \pm 12$ & $197 \pm 4.3$ \\
\hline NGC 4291 & 17.3 & BSG/JS & 12.32 & $30.3 \pm 3.7$ & $187 \pm 18$ & $200 \pm 6.5$ \\
\hline NGC 4472 & 104 & $\mathrm{SBS} / \mathrm{SZ} / \mathrm{WF}$ & 9.26 & $13.33 \pm 0.71$ & $198 \pm 9.2$ & $261 \pm 14$ \\
\hline NGC 4486B & $(3.07)^{a}$ & BSG & 14.26 & $13.33 \pm 0.71$ & $167 \pm 8.3$ & $190 \pm 7.1$ \\
\hline NGC 4486 & 94.9 & WF & 9.47 & $13.33 \pm 0.71$ & $238 \pm 12$ & $(265 \pm 2.6)^{d}$ \\
\hline NGC 4494 & 48.7 & JS & 10.61 & $7.0 \pm 1.5$ & $127 \pm 21$ & $135 \pm 8.2$ \\
\hline NGC 4564 & 19.8 & BSG & 11.91 & $13.33 \pm 0.71$ & $80 \pm 10$ & $105 \pm 10$ \\
\hline NGC 4621 & 40.5 & Bender & 10.5 & $13.33 \pm 0.71$ & $140 \pm 40$ & $210 \pm 10$ \\
\hline NGC 4660 & 12.2 & BSG & 12.12 & $13.33 \pm 0.71$ & $110 \pm 10$ & $135 \pm 7.1$ \\
\hline NGC 5018 & 22.8 & $\mathrm{CQ}$ & 11.34 & $29.8 \pm 3.1$ & $210 \pm 7.1$ & $215 \pm 14$ \\
\hline NGC 5576 & 18.1 & BSG & 11.71 & $16.5 \pm 2.5$ & $100 \pm 20$ & $185 \pm 10$ \\
\hline NGC 5812 & 25.5 & $\mathrm{BZ}$ & 11.87 & $21.1 \pm 4.5$ & $173 \pm 117$ & $175 \pm 17$ \\
\hline NGC 6411 & 28.6 & JS & 12.64 & $27.8 \pm 5.9$ & $333 \pm 120$ & $167 \pm 15$ \\
\hline NGC 7144 & 32.1 & $\mathrm{SZ}$ & 11.68 & $18.4 \pm 2.7$ & $155 \pm 14$ & $145 \pm 15$ \\
\hline NGC 7454 & 25.0 & BSG & 12.7 & $16.2 \pm 3.4$ & $97.7 \pm 8.3$ & $130 \pm 10$ \\
\hline NGC 7507 & 30.7 & $\mathrm{BZ}$ & 11.28 & $17.5 \pm 3.7$ & $180 \pm 15$ & $207 \pm 10$ \\
\hline NGC 7626 & 38.6 & JS & 12.06 & $35.8 \pm 3.8$ & $166 \pm 86$ & $208 \pm 17$ \\
\hline NGC 7785 & 23.3 & BSG & 12.41 & $45.0 \pm 9.6$ & $210 \pm 30$ & $192 \pm 8.9$ \\
\hline NGC 7796 & 21.2 & $\mathrm{BZ}$ & 12.39 & $(32.9 \pm 0.24)^{b}$ & $225 \pm 37$ & $233 \pm 12$ \\
\hline IC 179 & 16.5 & JS & 12.98 & $44.0 \pm 3.8$ & $124 \pm 21$ & $171 \pm 8.7$ \\
\hline
\end{tabular}

${ }^{a}$ This value is from BSG.

${ }^{b}$ The local peculiar velocity contributes to the distance estimate in RC3.

${ }^{c}$ Reference WF (Winsall \& Freeman 1993) is not used.

$d$ This value is from DB.

Table 1

The galaxy sample used in deriving the velocity dispersion-luminosity relation. The names of galaxies are listed in the first column. Their corresponding effective radii, observational sources, apparent magnitudes, distances, velocity dispersions at effective radii, and velocity dispersions at 0.54 effective radii are listed in columns 2 to 7 , respectively. 